\title{
Canonical Variate Dissimilarity Analysis for Process Incipient Fault Detection
}

\author{
Karl Ezra S. Pilario and Yi Cao, Senior Member, IEEE
}

\begin{abstract}
Early detection of incipient faults in industrial processes is increasingly becoming important, as these faults can slowly develop into serious abnormal events, an emergency situation, or even failure of critical equipment. Multivariate statistical process monitoring methods are currently established for abrupt fault detection. Among these, canonical variate analysis (CVA) was proven to be effective for dynamic process monitoring. However, the traditional CVA indices may not be sensitive enough for incipient faults. In this work, an extension of CVA, called the canonical variate dissimilarity analysis (CVDA), is proposed for process incipient fault detection in nonlinear dynamic processes under varying operating conditions. To handle non-Gaussian distributed data, kernel density estimation was used for computing detection limits. A CVA dissimilarity-based index has been demonstrated to outperform traditional CVA indices and other dissimilarity-based indices, namely DISSIM, RDTCSA, and GCCA, in terms of sensitivity when tested on slowly developing multiplicative and additive faults in a CSTR under closed-loop control and varying operating conditions.
\end{abstract}

\section{Index Terms}

Canonical variate analysis, kernel density estimation, dissimilarity analysis, nonlinear dynamic process.

Manuscript received October 03, 2017; revised December 13, 2017 and February 01, 2018; accepted February 24, 2018. Manuscript received in final form Month xx, 2018. The work of K.E.S. Pilario is supported by the DOST-ERDT Faculty Development Fund of the Republic of the Philippines while at Cranfield University, U.K., Paper no. TII-17-2341 (Corresponding author: K.E.S. Pilario).

The authors are with the School of Water, Energy, and Environment, Cranfield University, MK43 0AL, U.K. (email: k.pilario@cranfield.ac.uk; yi4cao2@gmail.com).

Color versions of one or more of the figures in this paper are available online at http://ieeexplore.ieee.org.

Digital Object Identifier: 
CSTR Continuous Stirred-tank Reactor

CV Canonical Variable

CVA Canonical Variate Analysis

CVDA

Canonical Variate Dissimilarity

Analysis

DD Detection Delay

DIS-

SIM

Dissimilarity Analysis

FAR False Alarm Rate

GCCA Generalized Canonical Correlation

Analysis

KDE Kernel Density Estimation

MDR Missed Detection Rate

MSPM

Multivariate Statistical Process

PCA Principal Components Analysis

PLS Partial Least Squares

Recursive Dynamic Transformed

RDTCSA

Component Statistical Analysis

SV Singular Value

SVD Singular Value Decomposition

UCL Upper Control Limit

\section{INTRODUCTION}

$\mathbf{I}$

NDUSTRIAL process monitoring deals with the detection, identification, diagnosis, and prognosis of abnormal events, or so-called faults, in industrial processes [1], [2]. A fault is formally defined as an "unpermitted deviation of at least one parameter of a process from acceptable conditions" [3]. More specifically, process monitoring methods aim to systematically [4]: (1) determine whether a fault has occurred (detection); (2) identify the process variables affected by the fault (identification); (3) determine the type, location, and magnitude of fault (diagnosis); and, (4) predict the evolution of a detected fault 
and its effects (prognosis). It is clear that these procedures are important for the safe, reliable, and environmentally benign operation of plants in any industry. Thus, automated industrial process monitoring has been an active field of study for decades now [1].

Widely used data-based fault detection methods, namely Multivariate Statistical Process Monitoring (MSPM) techniques, distinguish faulty from normal process states by establishing statistical thresholds around feature variables extracted from the process historical data [4]. Currently, MSPM techniques are being developed for nonlinear dynamic processes with the goals of increased sensitivity, early detection, and minimal false alarms during normal operation. Notable approaches are those based on principal components analysis (PCA) [5]-[8], partial least squares (PLS) [9], [10], independent component analysis [11], [12], and canonical variate analysis (CVA) [13], [14]. When compared to each other using benchmark case studies such as the Tennessee Eastman plant [4], [13], [15] and a multiphase flow facility [16], CVA-based methods were shown to be advantageous for nonlinear dynamic process monitoring in terms of detection rates and false alarm rates.

However, most existing literature focused on detecting abrupt faults. Isermann [3] distinguished between three types of fault according to how it progresses in time: abrupt, intermittent, and incipient. Abrupt faults are stepwise changes; intermittent faults are random occasional changes; while incipient faults are slowly developing changes. Early detection of incipient faults is more important than detection of abrupt faults for a number of reasons. When left unmanaged, incipient faults increase in severity, leading to more serious abnormal conditions. Even worse, it can lead to equipment failure or an emergency situation [17]. Thus, early detection is valuable for preventive maintenance of equipment and safer operations. Moreover, incipient faults require prognosis [18]. If a fault is detected in its incipient stage, operators may still decide to continue production; then, predictive monitoring can aid in subsequent production planning and provide lead time information before failure of critical assets occur [17], [19].

Unfortunately, incipient faults are more difficult to detect than abrupt faults. At the onset, they may be small enough to be accommodated by robust process control [20] or masked by noise, disturbances, or other faults. Thus, the aforementioned MSPM tools and their detection indices may not be sensitive enough to detect incipient faults.

To address this issue, Harmouche et al. [21] proposed PCA with kernel density estimation (KDE) and observed subtle differences in the estimated probability distribution of the principal components using the Kullback-Leibler divergence. The method was able to estimate the fault magnitude even when it is buried in noise. In a similar work, Harrou et al. [22] used the Hellinger distance metric on the nonlinear PLS algorithm as a measure of dissimilarity between the probability distribution of the latent variables. Promising results for incipient faults in a simulated plug flow reactor were presented. Meanwhile, Kano et 
al. [15], [23] proposed dissimilarity analysis on data sets (DISSIM), which also observed subtle changes in the data distribution structure. In DISSIM, the differences between a set of eigenvalues of two data sets are the features being monitored. In a recent study, Zhao et al. [24] extended the DISSIM algorithm for online incipient fault diagnosis. A recent MSPM technique called Recursive Dynamic Transformed Component Statistical Analysis was also proposed by Shang and Chen [25]. Here, orthogonal transformed components from a sliding window of measurements are obtained recursively. Similar to DISSIM, small changes in the higher-order statistics of the projected data are quantified to form a sensitive index, which was shown to be more reliable than DISSIM and dynamic PCA. However, these dissimilarity-based techniques require a large window width of samples for computing statistical patterns in the data. Also, they do not consider system dynamics, which is essential for monitoring under varying operating conditions.

On a different perspective, Ge et al. [26] used wavelet analysis combined with residual evaluation to extract an incipient fault feature, and used an improved KDE to form the thresholds. This method, however, requires additional steps to select wavelet basis functions and to tune various parameters so as to achieve acceptable false alarm and missed detection rates. Motivated by the fact that smoothing models such as the moving average and exponentially weighted moving average can detect small shifts in data, Ji et al. [27] proposed to combine them with the conventional MSPM tools, and devised novel generic fault detection indices. Here, "generic" means that the new indices can be applied to any MSPM model of quadratic form. Results have shown increased sensitivity, but only at certain smoothing parameters and weighting factors. Still, none of the above accounted for system dynamics.

To address the difficulty of detecting incipient faults for the case of nonlinear dynamic processes under varying operating conditions, this paper proposes Canonical Variate Dissimilarity Analysis (CVDA). Here, traditional CVA [13], [18] is extended to form a new index based on the dissimilarity between the pastprojected and future-projected canonical variables, called the canonical variate dissimilarity (CVD) index. CVDA retains the ability of CVA to handle the dynamic issue, as well as non-Gaussianity in the data by using KDE to compute detection limits. Using a closed-loop nonlinear process case study, namely a continuous stirred-tank reactor (CSTR), the CVD index was shown to be superior to the traditional CVA $T^{2}$ and $Q$ indices and also to other recent dissimilarity-based indices, in terms of detection time and reliability. This remains true for both multiplicative and additive incipient fault scenarios considered in this work.

In the next section, the procedure for traditional CVA monitoring is revisited. Section III discusses the motivation behind CVDA and the methodology. Section IV contains the case study description, results, and discussion. The paper is concluded in Section V, along with intended future work. 


\section{Canonical Variate Analysis ReVisited}

CVA is a linear dimensionality reduction technique that finds the maximum correlation between any two data sets [4]. Applications of CVA to industrial processes was pioneered by Larimore [28], [29]. As an MSPM tool, the data sets that CVA aims to correlate maximally are the past and future data during normal operation. Details about this method are as follows.

\section{A. CVA Training}

Negiz and Cinar [30] established that because industrial processes consist of a large number of process variables operating at controlled conditions, it is useful to assume that the process being monitored has a state-space realization, which is equivalent to representing the system in a vector autoregressive movingaverage time-series model with exogenous inputs. In linear state-space form, this model can be written as

$$
\begin{gathered}
\mathbf{x}(t+1)=\mathbf{A x}(t)+\mathbf{B u}(t)+\mathbf{K} \nu(t) \\
\mathbf{y}(t)=\mathbf{C x}(t)+\mathbf{D u}(t)+\nu(t)
\end{gathered}
$$

where $\mathbf{A}, \mathbf{B}, \mathbf{C}, \mathbf{D}$ and $\mathbf{K}$ are coefficient matrices, $\mathbf{x}(t)$ is the state vector, $\mathbf{y}(t)$ is the output observation vector, $\mathbf{u}(t)$ is the input vector, and $\nu(t)$ are independent noises.

Let $\mathbf{u}_{k} \in \Re^{m_{u}}$ and $\mathbf{y}_{k} \in \Re^{m_{y}}$ respectively denote the input and output column vectors from the process at a certain time point $k$. For each $k$, the past data vectors $\mathbf{p}_{k}$ and future data vectors $\mathbf{f}_{k}$ are collected as

$$
\begin{aligned}
& \mathbf{p}_{k}=\left[\begin{array}{llll}
\mathbf{u}_{k-1}^{T} & \mathbf{u}_{k-2}^{T} & \ldots & \mathbf{u}_{k-p}^{T}
\end{array}\right. \\
& \mathbf{f}_{k}=\left[\begin{array}{lllll}
\mathbf{y}_{k}^{T} & \mathbf{y}_{k+1}^{T} & \mathbf{y}_{k+2}^{T} & \ldots & \mathbf{y}_{k+f-1}^{T}
\end{array}\right]^{T} \in \Re^{m_{y} f}
\end{aligned}
$$

where $m=m_{u}+m_{y}$, and $p$ and $f$ are the number of lags considered in the past and future windows of data, respectively. The amount of lag is chosen large enough to capture data autocorrelation. The input vectors, $\mathbf{u}$, are excluded from the future data vectors considering their statistical independence from the past. Both data vectors are then normalized to zero mean and unit variance.

For a training set with $N$ number of observations, $\mathbf{p}_{k}$ and $\mathbf{f}_{k}$ for all $k \in[p+1, p+M]$ are appended column-wise to form the past and future Hankel matrices

$$
\begin{aligned}
& \mathbf{Y}_{p}=\left[\begin{array}{llll}
\mathbf{p}_{p+1} & \mathbf{p}_{p+2} & \ldots & \mathbf{p}_{p+M}
\end{array}\right] \in \Re^{m p \times M} \\
& \mathbf{Y}_{f}=\left[\begin{array}{llll}
\mathbf{f}_{p+1} & \mathbf{f}_{p+2} & \ldots & \mathbf{f}_{p+M}
\end{array}\right] \in \Re^{m_{y} f \times M}
\end{aligned}
$$

where $M=N-p-f+1$. 
The sample covariance and cross-covariance of the past and future observations can be estimated as

$$
\begin{gathered}
\boldsymbol{\Sigma}_{p p}=\frac{1}{M-1} \mathbf{Y}_{p} \mathbf{Y}_{p}^{T} \in \Re^{m p \times m p} \\
\boldsymbol{\Sigma}_{f f}=\frac{1}{M-1} \mathbf{Y}_{f} \mathbf{Y}_{f}^{T} \in \Re^{m_{y} f \times m_{y} f} \\
\boldsymbol{\Sigma}_{f p}=\frac{1}{M-1} \mathbf{Y}_{f} \mathbf{Y}_{p}^{T} \in \Re^{m_{y} f \times m p} .
\end{gathered}
$$

For training, CVA aims to find linear combinations of $\mathbf{Y}_{p}$ and $\mathbf{Y}_{f}$ with the maximum correlation. This can be achieved by a singular value decomposition (SVD) on the scaled Hankel matrix, $\mathbf{H}$ :

$$
\mathbf{H}=\boldsymbol{\Sigma}_{f f}^{-1 / 2} \boldsymbol{\Sigma}_{f p} \boldsymbol{\Sigma}_{p p}^{-1 / 2}=\mathbf{U} \boldsymbol{\Sigma} \mathbf{V}^{T}
$$

where $\mathbf{U}$ and $\mathbf{V}$ contains the left and right singular column vectors of $\mathbf{H}$, and $\boldsymbol{\Sigma}$ is a diagonal matrix of ordered singular values (SVs), i.e. $\boldsymbol{\Sigma}=\operatorname{diag}\left(\sigma_{1}, \sigma_{2}, \ldots, \sigma_{r}, 0, \ldots, 0\right)$ where $r$ is the rank of $\mathbf{H}$ and $1 \geq \sigma_{1} \geq \sigma_{2} \geq \ldots \geq \sigma_{r}$.

Projection matrices, $\mathbf{J}=\mathbf{V}^{T} \boldsymbol{\Sigma}_{p p}^{-1 / 2}$ and $\mathbf{L}=\mathbf{U}^{T} \boldsymbol{\Sigma}_{f f}^{-1 / 2}$, are now used to transform $\mathbf{Y}_{p}$ and $\mathbf{Y}_{f}$ to reveal maximally correlated canonical variables, $\mathcal{C}_{p}$ and $\mathcal{C}_{f}$, projected from the past and future data, respectively:

$$
\begin{gathered}
\mathcal{C}_{p}=\mathbf{J} \mathbf{Y}_{p} \in \Re^{m p \times M} \\
\mathcal{C}_{f}=\mathbf{L} \mathbf{Y}_{f} \in \Re^{m_{y} f \times M} .
\end{gathered}
$$

These canonical variables (CVs) are the features used to distinguish normal from faulty process states. At normal operation, the distributions of $\mathcal{C}_{p}$ and $\mathcal{C}_{f}$ are preserved.

\section{B. CVA Monitoring}

Important properties of the SVD in (10) are written below, showing the cross-covariance and covariance of the CVs:

$$
\begin{array}{r}
\mathcal{C}_{f} \mathcal{C}_{p}^{T}=\mathbf{L} \boldsymbol{\Sigma}_{f p} \mathbf{J}^{T}=\mathbf{\Sigma} \cdot(M-1) \\
\mathcal{C}_{p} \mathcal{C}_{p}^{T}=\mathbf{J} \boldsymbol{\Sigma}_{p p} \mathbf{J}^{T}=\mathbf{I} \cdot(M-1) \\
\mathcal{C}_{f} \mathcal{C}_{f}^{T}=\mathbf{L} \boldsymbol{\Sigma}_{f f} \mathbf{L}^{T}=\mathbf{I} \cdot(M-1) .
\end{array}
$$

Equation (13) indicates that the SVs, $\sigma_{i}(i=1,2, \ldots, r)$, are the canonical correlations of the CVs. Based on the SV trend, there exists only $n(<r)$ strongly correlated CVs which explain most of the system dynamic behavior. Hence, the CV space can be partitioned into two orthogonal subspaces. The first $n$ CVs span the state subspace, $\mathbf{Z}$, of the system in (1). In other words, the first $n$ CVs at time $t$, $\mathbf{z}_{t}$, represent the state variables of the system, i.e. $\mathbf{x}(t)=\mathbf{z}_{t}$ [28]. Likewise, the remaining CVs span the 
system residual subspace, E. In traditional CVA-based strategies [4], [13], only the past data vectors are used to reveal the state and residual subspaces:

$$
\begin{gathered}
\mathbf{z}_{k}=\mathbf{J}_{n} \mathbf{p}_{k} \in \Re^{n} \\
\mathbf{e}_{k}=\mathbf{F} \mathbf{p}_{k} \in \Re^{m p}
\end{gathered}
$$

where $\mathbf{J}_{n}=\mathbf{V}_{n}^{T} \boldsymbol{\Sigma}_{p p}^{-1 / 2}, \mathbf{F}=\left(\mathbf{I}-\mathbf{V}_{n} \mathbf{V}_{n}^{T}\right) \boldsymbol{\Sigma}_{p p}^{-1 / 2}, \mathbf{z}_{k}$ is the state vector at time $k, \mathbf{e}_{k}$ is the model residual vector at time $k$, and $\mathbf{V}_{n}$ is a reduced matrix consisting of the first $n$ columns of $\mathbf{V}$. Matrices $\mathbf{J}_{n}$ and $\mathbf{F}$ represent the projection matrices applied to the past data vectors.

Hotelling's $T^{2}$ and the $Q$ statistics, two widely used indices in process monitoring, are computed at each time point $k$ :

$$
\begin{gathered}
T_{k}^{2}=\mathbf{z}_{k}^{T} \mathbf{z}_{k} \\
Q_{k}=\mathbf{e}_{k}^{T} \mathbf{e}_{k} .
\end{gathered}
$$

Upper control limits (UCLs) for these two indices can be obtained analytically by assuming that the underlying process data follows a Gaussian distribution. However, this is no longer valid for nonlinear processes under varying operating conditions. To address this, kernel density estimation (KDE) is used to estimate the probability distributions of $T^{2}$ and $Q$ [13]. The chosen kernel in this paper is the radial basis function:

$$
K(g)=\frac{1}{\sqrt{2 \pi}} \exp \left(-\frac{g^{2}}{2}\right) .
$$

The UCLs serve as thresholds between normal and abnormal conditions. Given a significance level, $\alpha$, the UCLs denoted by $T_{\mathrm{UCL}}^{2}$ and $Q_{\mathrm{UCL}}$ can be obtained such that $P\left(T^{2}<T_{\mathrm{UCL}}^{2}\right)=\alpha$ and $P(Q<$ $\left.Q_{\mathrm{UCL}}\right)=\alpha$, with

$$
P(x<b)=\int_{-\infty}^{b} \frac{1}{M h} \sum_{k=1}^{M} K\left(\frac{x-x_{k}}{h}\right) d x
$$

where $x_{k}, k=1,2, \cdots, M$ are the samples of $x$ and $h$ is the kernel bandwidth. More details for KDE are given in [13].

Finally, for online monitoring, the state and residuals are continuously calculated from real-time data using (16) and (17). The $T^{2}$ and $Q$ at every sampling point are then obtained using (18) and (19). A fault is detected when any one of these indices exceed its corresponding UCL, $T_{\mathrm{UCL}}^{2}$ or $Q_{\mathrm{UCL}}$, respectively. 


\section{CANONICAL VARIATE Dissimilarity Analysis}

Process incipient faults manifest as small shifts in the data caused by decay in process parameters, unwanted material build-up, or sensor drifts, etc. Motivated by the ability of CVA to find maximum correlations between past and future data, one can detect small data shifts by checking how well future $\mathrm{CVs}$ are predictable from past $\mathrm{CVs}$, based on the state-space model trained during normal conditions. This leads to the notion of assessing the dissimilarity between the past- and future-projected CVs for indicating process health.

A statistical index that quantifies model residuals in the CVA state-subspace has been suggested by Larimore [29]. In that work, the residuals, $\epsilon$, are generated as

$$
\boldsymbol{\epsilon}_{k}=\mathbf{L}_{n} \mathbf{f}_{k}-\boldsymbol{\Sigma}_{n} \mathbf{J}_{n} \mathbf{p}_{k} \in \Re^{n}
$$

where subscript $k$ denotes the $k$ th sample, subscript $n$ denotes the number of states in the CVA model (which reduces $\mathbf{J}$ and $\mathbf{L}$ to the first $n$ rows), and $\boldsymbol{\Sigma}_{n}=\operatorname{diag}\left(\sigma_{1}, \sigma_{2}, \ldots, \sigma_{n}\right)$. To our knowledge, the $T_{\epsilon}^{2}$ index formed from the residuals in (22) is underexplored among numerous CVA-based fault detection methods. In one study, Juricek et al. [31] found that an index similar to (22) outperforms a Kalman filter residuals-based index with respect to sensitivity, although their index requires state-space matrices to be calculated, hence is not data-driven. Analogous formulations of (22) are also found in recent works by Jiang et al. [32] and Chen et al. [33] except that canonical correlation analysis was applied between process inputs and outputs instead of past and future data. For our work, the residuals in (22) are taken as dissimilarity features which can have a new meaning for detecting incipient faults. These features are measures of the departure of the current actual states (estimated from the future vector that includes current measurements) from the states predicted by the CVA model using past data.

To derive an index from (22), let $\mathbf{d}$ denote the dissimilarity features, i.e. $\mathbf{d}:=\boldsymbol{\epsilon}$. Also, let $\mathbf{Z}_{f}=\mathbf{L}_{n} \mathbf{Y}_{f}$ and $\mathbf{Z}_{p}=\mathbf{J}_{n} \mathbf{Y}_{p}$ denote the future- and past-projected states, respectively. Since $\mathbf{Z}_{f} \mathbf{Z}_{f}^{T}=\mathbf{Z}_{p} \mathbf{Z}_{p}^{T}=\mathbf{I}$ and $\mathbf{Z}_{f} \mathbf{Z}_{p}^{T}=\Sigma_{n}$, the covariance of $\mathbf{d}$ can be estimated as

$$
\begin{aligned}
\boldsymbol{\Sigma}_{\mathbf{d d}} & =\frac{1}{M-1}\left(\mathbf{Z}_{f}-\boldsymbol{\Sigma}_{n} \mathbf{Z}_{p}\right)\left(\mathbf{Z}_{f}-\boldsymbol{\Sigma}_{n} \mathbf{Z}_{p}\right)^{T} \\
& =\mathbf{I}-\boldsymbol{\Sigma}_{n}^{2} \in \Re^{n \times n} .
\end{aligned}
$$

At normal operation, the dissimilarity features are distributed around a zero mean. Thus, a detection index can be formed as the squared Mahalanobis distance of the dissimilarity features from zero, i.e. by taking the sum of squares of the dissimilarities $\mathbf{d}_{k} \in \Re^{n}$, for each time point $k$, normalized by the covariance matrix, $\Sigma_{\mathrm{dd}}$ :

$$
D_{k}=\mathbf{d}_{k}^{T}\left(\mathbf{I}-\Sigma_{n}^{2}\right)^{-1} \mathbf{d}_{k} .
$$




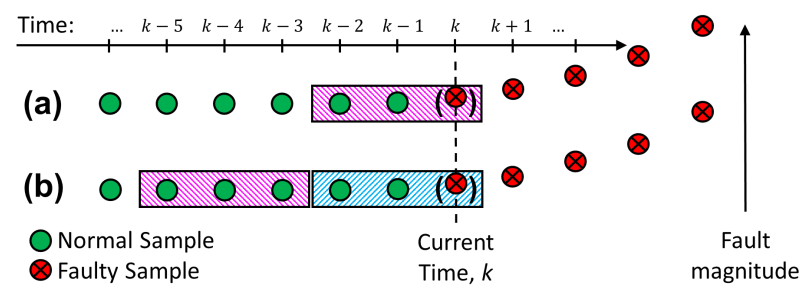

Fig. 1. Initial stage of a fault and how it is captured by (a) traditional CVA indices; and (b) CVD index. Legend: past vector - pink; future vector - blue.

Hereafter, $D$ is referred to as the canonical variate dissimilarity (CVD) index. Unlike the CVA $T^{2}$ and $Q$ indices in (18)-(19) which only take information from the past data, the CVD index takes information from both past and future data. For online monitoring using CVA, each new sample enters only the past data vector of length $p$. On the other hand, for CVD, each new sample enters the future data vector, while the past data vector is formed from $p$ samples, $f$ lags earlier. Thus, in the early stage of an incipient fault shown in Fig. 1 (where $p=f=3$ ), small shifts can be better captured as discrepancies found between the past and future data.

In this paper, KDE is adopted to estimate the distribution of $D$, and subsequently solve for the upper control limit of $D$, denoted by $D_{\mathrm{UCL}}$. All three indices $T^{2}, Q$, and $D$, will be used for monitoring incipient faults in nonlinear dynamic processes under the framework referred to as canonical variate dissimilarity analysis (CVDA). A sample is considered faulty if either $T^{2}, Q$, or $D$, exceeds their respective thresholds, $T_{\mathrm{UCL}}^{2}, Q_{\mathrm{UCL}}$, and $D_{\mathrm{UCL}}$, for that sample.

In summary, the CVDA procedure for incipient fault detection is given in Fig. 2. The goal of offline training is to generate the UCLs and the projection matrices. On the other hand, online monitoring consists of the continuous collection of a moving window of samples of length $p+f$ (Fig. 1b), which are used to check if the process is normal or faulty.

\section{CASe Study}

In this section, the proposed CVDA is evaluated using a closed-loop continuous stirred-tank reactor (CSTR) case study, designed especially for simulating incipient faults.

\section{A. Process Description}

A number of works, such as [6], [8], [10], [17], [18], [31], used different versions of a CSTR for evaluating process monitoring methods. A general first-principles model of a constant-holdup, jacketed tank that continuously carries out an exothermic first-order reaction, $A \rightarrow B$, is given as 


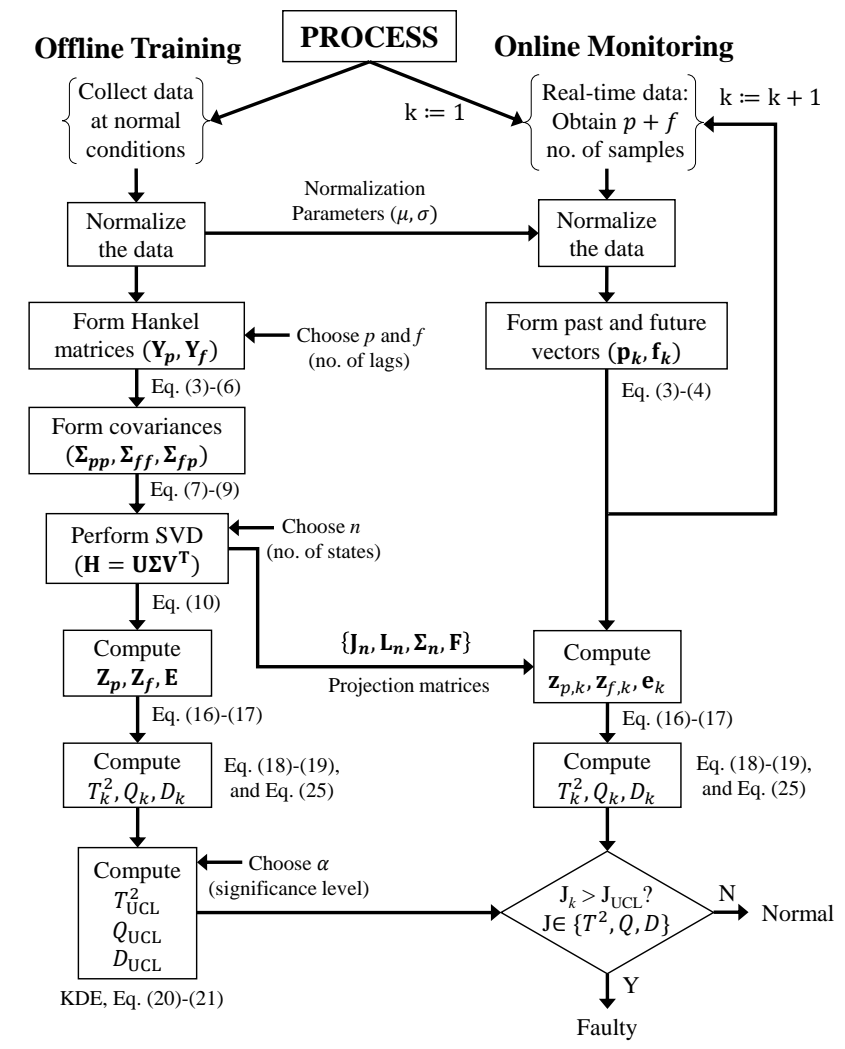

Fig. 2. CVDA procedure for process incipient fault detection.

$$
\begin{gathered}
\frac{d C}{d t}=\frac{Q}{V}\left(C_{i}-C\right)-a k C+\nu_{1} \\
\frac{d T}{d t}=\frac{Q}{V}\left(T_{i}-T\right)-a \frac{\left(\Delta H_{r}\right) k C}{\rho C_{p}}-b \frac{U A}{\rho C_{p} V}\left(T-T_{c}\right)+\nu_{2} \\
\frac{d T_{c}}{d t}=\frac{Q_{c}}{V_{c}}\left(T_{c i}-T_{c}\right)+b \frac{U A}{\rho_{c} C_{p c} V_{c}}\left(T-T_{c}\right)+\nu_{3}
\end{gathered}
$$

where the inputs are $\mathbf{u}=\left[\begin{array}{lll}C_{i} & T_{i} & T_{c i}\end{array}\right]^{T}$, the outputs are $\mathbf{y}=\left[\begin{array}{llll}C & T & T_{c} & Q_{c}\end{array}\right]^{T}, \nu_{i}$ are process noise, and $k$ is an Arrhenius-type rate constant, $k=k_{0} \exp \left(\frac{-E}{R T}\right)$. Table I gives the parameter values in Eq. (26)-(28). The CSTR Simulink model used in this work is available online [34].

The CSTR schematic in Fig. 3 shows the measurement locations and the control strategy: Reactor temperature, $T$, is maintained by manipulating the coolant flow rate, $Q_{c}$. To be more realistic, the controller ( $K_{c}=1.0$ and $\tau_{I}=0.2$ ) is set to saturate below $10 \mathrm{~L} / \mathrm{min}$ and above $200 \mathrm{~L} / \mathrm{min}$. Saturation is important for simulating cases where an incipient fault becomes too severe to be accommodated by controls. In the model, $a$ and $b$ are both equal to 1.00 at normal operation. By decaying their values toward zero, one can simulate catalyst decay and heat transfer fouling, respectively. Other faults in the system are sensor drifts on each of the 7 measured variables. Details on these incipient fault scenarios are given in Table II. 
TABLE I

CONSTANT VALUES IN THE CSTR MODEL

\begin{tabular}{cccc}
\hline Parameter & Description & Value & Units \\
\hline$Q$ & Inlet flow rate & 100.0 & $\mathrm{~L} / \mathrm{min}$ \\
$V$ & Tank volume & 150.0 & $\mathrm{~L}$ \\
$V_{c}$ & Jacket volume & 10.0 & $\mathrm{~L}$ \\
$\Delta H_{r}$ & Heat of reaction & $-2.0 \times 10^{5}$ & $\mathrm{cal} / \mathrm{mol}$ \\
$U A$ & Heat transfer coefficient & $7.0 \times 10^{5}$ & $\mathrm{cal} / \mathrm{min} / \mathrm{K}$ \\
$k_{0}$ & Pre-exponential factor to $k$ & $7.2 \times 10^{10}$ & $\mathrm{~min}{ }^{-1}$ \\
$E / R$ & Activation energy & $1.0 \times 10^{4}$ & $\mathrm{~K}$ \\
$\rho, \rho_{c}$ & Fluid density & 1000 & $\mathrm{~g} / \mathrm{L}$ \\
$C_{p}, C_{p c}$ & Fluid heat capacity & 1.0 & $\mathrm{cal} / \mathrm{g} / \mathrm{K}$ \\
\hline
\end{tabular}

TABLE II

INCIPIENT FAULT SCENARIOS IN THE CSTR

\begin{tabular}{cccc}
\hline Fault ID & Description $^{\dagger}$ & Value of $\delta^{\ddagger}$ & Type \\
\hline 1 & $a=a_{0} \exp (-\delta t)$ & 0.0005 & Multiplicative \\
2 & $b=b_{0} \exp (-\delta t)$ & 0.001 & Multiplicative \\
3 & Simultaneous Faults 1 and 2 & & Multiplicative \\
4 & $C_{i}=C_{i, 0}+\delta t$ & 0.001 & Additive \\
5 & $T_{i}=T_{i, 0}+\delta t$ & 0.05 & Additive \\
6 & $T_{c i}=T_{c i, 0}+\delta t$ & 0.05 & Additive \\
7 & $C=C_{0}+\delta t$ & 0.001 & Additive \\
8 & $T=T_{0}+\delta t$ & 0.05 & Additive \\
9 & $T_{c}=T_{c, 0}+\delta t$ & 0.05 & Additive \\
10 & $Q_{c}=Q_{c, 0}+\delta t$ & -0.1 & Additive \\
\hline
\end{tabular}

${ }^{\dagger}$ All $t$ in minutes. Subscript 0 refers to nominal value.

$\ddagger$ The $\delta$ commands the rate of fault progression.

Fault-free and faulty data sets were generated from the CSTR simulation for $20 \mathrm{~h}$ of operation, while varying the operating conditions by perturbing the inputs, $\mathbf{u}$, randomly around their nominal values every $60 \mathrm{~min}$ as in Fig. 4. The sampling interval for all variables is $1 \mathrm{~min}$. Note that input disturbances can bring out system dynamics, such that measurements become temporally correlated and non-Gaussian-distributed due to the process nonlinearity. This merits the use of CVA and KDE for the CSTR case study, as these are 


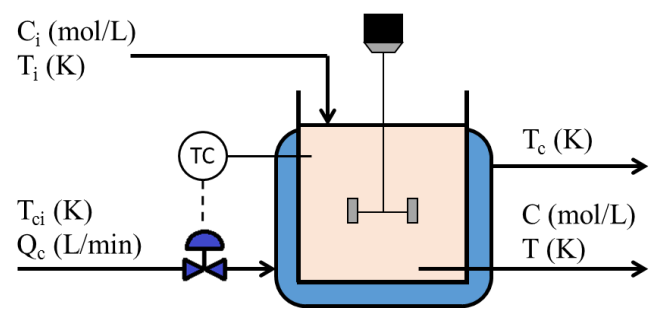

Fig. 3. Schematic of the closed-loop CSTR.
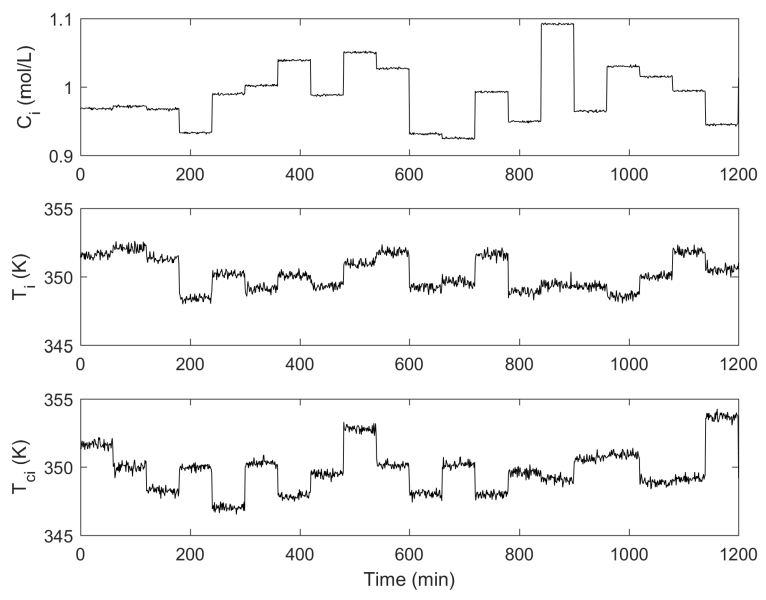

Fig. 4. Input variables sample data set from CSTR simulation.

recommended for nonlinear dynamic process monitoring [13]. In faulty data sets, the fault is introduced only after $200 \mathrm{~min}$ of normal operation. To evaluate the performance robustly, 250 faulty data sets are generated in each fault scenario, differing in the random seeds for the process noise, measurement noise, and input disturbances. Performance metrics for monitoring (see Section IVc) are averaged across all trials.

\section{B. CVDA Training}

A single fault-free data set was used for CVDA training. Firstly, the number of time lags to consider in the past and future data ( $p$ and $f$ ) were chosen using autocorrelation analysis [30] on the output training data, $\mathbf{y}$. For the case study, it was found that five number of lags is the maximum after which autocorrelations in data become insignificant at $5 \%$ confidence bounds. Thus, $p$ and $f$ are both set to 5 .

SVD was then performed on the scaled Hankel matrix, which was formed based on $p$ and $f$. The number of states, $n$, in the CVA model was then estimated from the training data. A plot of the singular values (SVs) computed from (10) is shown in Fig. 5a, which are the non-zero elements in the diagonal matrix, $\Sigma$. One can estimate $n$ to be the point where a "knee" appears in the SV curve [30]. This is 

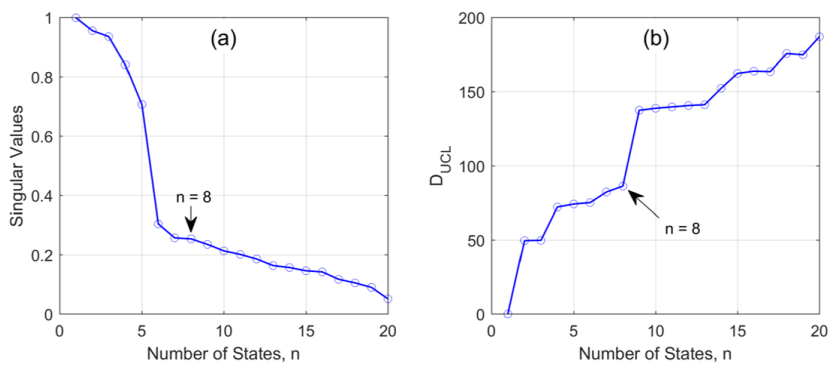

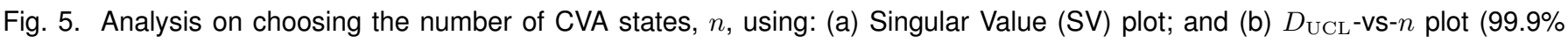
significance level).

commonly called the dominant singular value method of selecting $n$. Other ways of selecting $n$ include using the Akaike Information Criterion [4] or minimizing false alarms during cross-validation [16].

In this work, we propose to use $D_{\mathrm{UCL}^{-}}-v s-n$ plots to augment the dominant singular value method in selecting $n$. If we proceed with the CVDA procedure until $D_{\mathrm{UCL}}$ is computed, we can produce a plot of $D_{\mathrm{UCL}}$ 's against increasing choices of $n$ (Fig. 5b). Intuitively, $D_{\mathrm{UCL}}$ measures how precisely the CVA model predicts the future state. For small values of $n$, prediction is precise because the dissimilarities between the first $n$ past and future CVs are small, i.e. $D_{\mathrm{UCL}}$ is low. For large $n$, the dissimilarities become large due to capture of weakly correlated CVs, i.e. $D_{\mathrm{UCL}}$ is high. Therefore, a large jump in the $D_{\mathrm{UCL}}$-vs- $n$ curve indicates a sudden entry of unnecessary noise into the model, i.e. the clear boundary between the state and residual subspaces. Figure $5 \mathrm{~b}$ shows the $D_{\mathrm{UCL}}-\mathrm{vs}-n$ plot for the CSTR training data. The point right before the jump in the curve is located at $n=8$. This choice agrees with the SV plot on Fig. 5a, where the "knee" appears at $n \geq 6$. Thus, for the case study, $n$ is set to 8. From here, the CVA model is now trained and the actual detection limits are computed using KDE at $99.9 \%$ significance level.

\section{CVDA Monitoring}

In this work, monitoring performance is evaluated according to detection delay (DD), false alarm rate (FAR), missed detection rate (MDR), and visually using monitoring charts (Fig. 6). Note that because we are monitoring slowly developing faults, detection time could be ambiguous, i.e. the index may spend a considerable amount of time fluctuating around the detection limit before it fully registers a fault. Thus, detection time is defined as the first time after 5 consecutive alarms were raised. Accordingly, DD is the period between the start of the fault and the detection time. FAR and MDR are computed as:

$$
\begin{gathered}
\mathrm{FAR}=\frac{\text { no. of samples }\left(J>J_{\mathrm{UCL}} \mid \text { fault-free }\right)}{\text { total samples }(\text { fault-free })} \times 100 \% \\
\mathrm{MDR}=\frac{\text { no. of samples }\left(J<J_{\mathrm{UCL}} \mid \text { fault }\right)}{\text { total samples (fault) }} \times 100 \%
\end{gathered}
$$



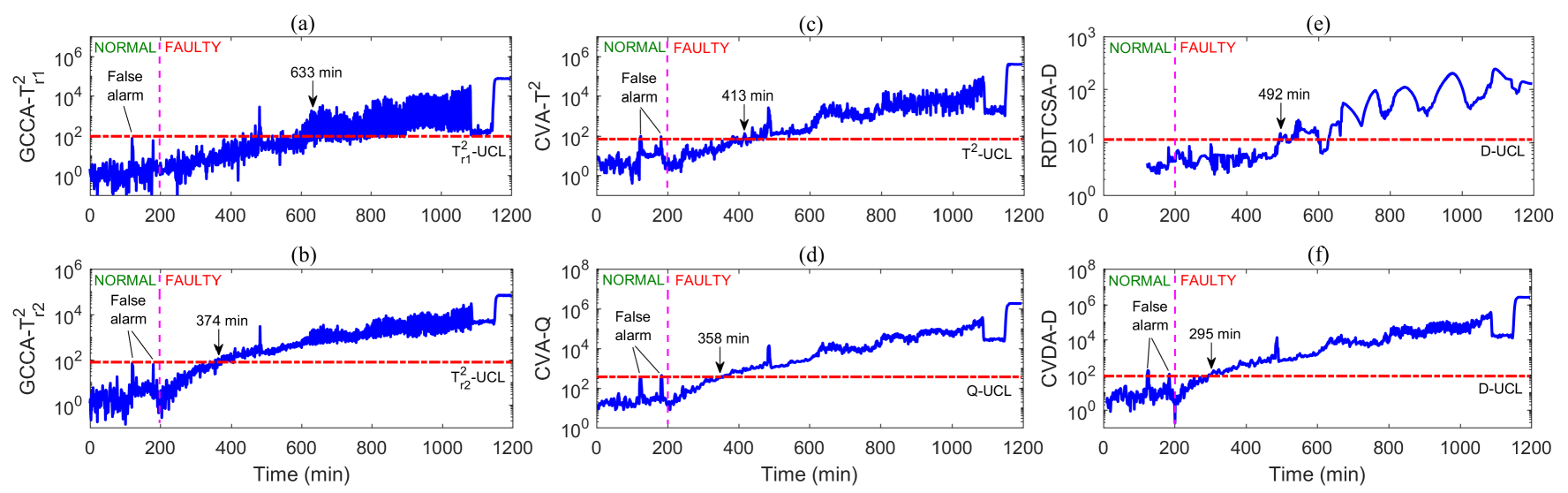

Fig. 6. Monitoring charts for Fault 3 using: (a,b) GCCA; (c,d) CVA; (e) RDTCSA; (f) CVDA. See Section IVc for implementation details. Legend: Dashdot - upper control limit (UCL); Dash - start of fault; Solid - detection index.

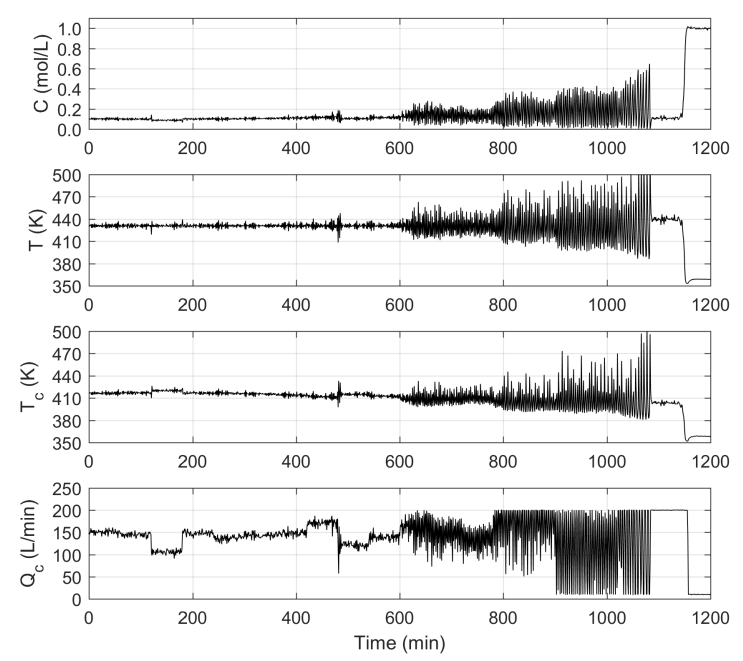

Fig. 7. Output variables sample data set under Fault 3 conditions: catalyst decay and heat transfer fouling were both introduced at $200 \mathrm{~min}$.

where $J=\left\{T^{2}, Q, D\right\}$. A good detection index must have low DD, low FAR, and low MDR. DD is particularly important for assessing the sensitivity of a detection index.

Table III compares the proposed CVD index with the traditional CVA indices [18] and other dissimilaritybased indices from recent works: dynamic DISSIM [23], Recursive Dynamic Transformed Component Statistical Analysis (RDTCSA) [25], and Generalized Canonical Correlation Analysis (GCCA) [33]. For the dynamic DISSIM and RDTCSA indices, five lagged variables (same as CVDA) and a window width of 120 samples were used. For RDTCSA, the sample mean, variance, skewness, and kurtosis are included in the feature vector, scalarized by the $\ell_{\infty}$-norm. For GCCA, both $T_{r 1}^{2}$ and $T_{r 2}^{2}$ were used as defined in [33], except that the UCL was not computed using randomized algorithms. Rather, all UCLs in this study were computed using KDE at 99.9\% significance level. Detection delays in Table III are reported 
in units of hours due to the slow rate of progression designed for the incipient faults (see Table II). This design also makes the distinction between methods more pronounced.

In terms of DD and MDR, the CVD index is seen to be most sensitive for all fault scenarios. The dynamic DISSIM and RDTCSA incurred non-detection for some faults, as they do not consider the system dynamics that can be inferred from the temporal correlation in the data. Although the RDTCSA index detected Fault 5 the earliest, the high MDR reveals that the index struggles to cross the detection limit. The GCCA indices, $T_{r 1}^{2}$ and $T_{r 2}^{2}$, are more reliable, as one of them is sensitive to the faults where the other is not (e.g. Faults 5 and 6). Consistent with the findings in [33], $T_{r 2}^{2}$ is often more sensitive than $T_{r 1}^{2}$. Also, the GCCA indices appear to incur the least false alarms during normal operation. Indeed, although the CVD index is most sensitive, it incurs slightly more false alarms. Nonetheless, the CVD index is most reliable in terms of combined FARs and MDRs. These results validate the importance of using the dissimilarity between past and future states for incipient fault detection, rather than past states only, as in traditional CVA.

To appreciate the performance of the CVD index, sample monitoring charts for Fault 3 are shown in Fig. 6, with the corresponding y data in Fig. 7. Fault 3 leads to conflicting behavior (at $600 \mathrm{~min}$ ) in the affected variables because the rates of catalyst decay and heat transfer fouling were set so as to keep opposing each other [18]. Eventually, failure occurs after 1085 min. Fig. 6f shows how the proposed CVD index achieves the earliest detection time among the indices being compared, at 295 min. This detection time may not be achieved by mere human inspection on Fig. 7. Thus, using this index, more lead time before failure is realized. Furthermore, it is beneficial for an index to properly reflect fault severity even as it progresses above the UCL. This trait is best observed in the CVD index as well.

The charts in Fig. 6 also marked false alarms. It was found that false alarms mostly occur at times when inputs change abruptly (Fig. 4), causing unrecognized transients in the data (Fig. 7). Thus, one way to lessen false alarms is to train a nonlinear model [14] for CVDA that may recognize these transients. This is left for future work.

\section{CONCLUSION}

In this paper, the importance of incipient fault detection, rather than abrupt fault, in industrial processes is highlighted. For dynamic process monitoring, the traditional CVA detection method is recognized. This work now extends the CVA framework to emphasize that the assessment of dissimilarity between past and future states gives a more sensitive index than the traditional $T^{2}$ and $Q$ for incipient fault detection. A method for subspace partitioning using $D_{\mathrm{UCL}}-\mathrm{vs}-n$ plots is also proposed. The new framework, called canonical variate dissimilarity analysis (CVDA), is intended for nonlinear dynamic process monitoring of incipient faults under varying process operating conditions. 
Using a CSTR case study designed to simulate ten slowly developing faults, the CVD index was shown to be superior to the CVA $T^{2}$ and $Q$ indices, the dynamic DISSIM, RDTCSA, and the GCCA indices in terms of sensitivity. However, the CVD index incurred more false alarms, mostly occurring when operating conditions change abruptly. This can be lessened by using a nonlinear feature extraction step for CVDA. Other ways to quantify statistical pattern dissimilarity between CVs also require further study. Lastly, diagnosis and prognosis methods from the CVDA framework can be developed for incipient fault monitoring in the future.

\section{REFERENCES}

[1] M. Reis and G. Gins, "Industrial Process Monitoring in the Big Data/Industry 4.0 Era: from Detection, to Diagnosis, to Prognosis," Processes, vol. 5, no. 3, p. 35, 2017.

[2] Y. Shu, L. Ming, F. Cheng, Z. Zhang, and J. Zhao, "Abnormal situation management: Challenges and opportunities in the big data era," Computers and Chemical Engineering, vol. 91, pp. 104-113, 2016.

[3] R. Isermann, "Model-based fault-detection and diagnosis - Status and applications," Annual Reviews in Control, vol. 29, no. 1, pp. 71-85, 2005.

[4] L. H. Chiang, E. L. Russell, and R. D. Braatz, Fault Detection and Diagnosis in Industrial Systems. London: Springer-Verlag, 2005.

[5] W. Ku, R. H. Storer, and C. Georgakis, "Disturbance detection and isolation by dynamic principal component analysis," Chemometrics and Intelligent Laboratory Systems, vol. 30, no. 1, pp. 179-196, 1995.

[6] S. W. Choi, C. Lee, J. M. Lee, J. H. Park, and I. B. Lee, "Fault detection and identification of nonlinear processes based on kernel PCA," Chemometrics and Intelligent Laboratory Systems, vol. 75, no. 1, pp. 55-67, 2005.

[7] R. T. Samuel and Y. Cao, "Nonlinear process fault detection and identification using kernel PCA and kernel density estimation," Systems Science \& Control Engineering, vol. 4, no. 1, pp. 165-174, 2016.

[8] M. Mansouri, M. Nounou, H. Nounou, and N. Karim, "Kernel PCA-based GLRT for nonlinear fault detection of chemical processes," Journal of Loss Prevention in the Process Industries, vol. 40, pp. 334-347, 2016.

[9] C. Shang, X. Huang, J. A. K. Suykens, and D. Huang, "Enhancing dynamic soft sensors based on DPLS: A temporal smoothness regularization approach,” Journal of Process Control, vol. 28, pp. 17-26, 2015.

[10] C. Botre, M. Mansouri, M. Nounou, H. Nounou, and M. N. Karim, "Kernel PLS-based GLRT method for fault detection of chemical processes," Journal of Loss Prevention in the Process Industries, vol. 43, pp. 212-224, 2016.

[11] J. Fan and Y. Wang, "Fault detection and diagnosis of non-linear non-Gaussian dynamic processes using kernel dynamic independent component analysis," Information Sciences, vol. 259, pp. 369-379, 2014.

[12] G. Stefatos and A. B. Hamza, "Dynamic independent component analysis approach for fault detection and diagnosis," Expert Systems with Applications, vol. 37, no. 12, pp. 8606-8617, 2010.

[13] P.-E. Odiowei and Yi Cao, "Nonlinear Dynamic Process Monitoring Using Canonical Variate Analysis and Kernel Density Estimations," IEEE Transactions on Industrial Informatics, vol. 6, no. 1, pp. 36-45, 2010.

[14] R. T. Samuel and Y. Cao, "Kernel canonical variate analysis for nonlinear dynamic process monitoring," IFAC-PapersOnLine, vol. 28, no. 8, pp. 605-610, 2015.

[15] M. Kano, K. Nagao, S. Hasebe, I. Hashimoto, H. Ohno, R. Strauss, and B. Bakshi, "Comparison of statistical process monitoring methods: application to the Eastman challenge problem," Computers \& Chemical Engineering, vol. 24, no. 2-7, pp. 175-181, 2000.

[16] C. Ruiz-Cárcel, Y. Cao, D. Mba, L. Lao, and R. T. Samuel, "Statistical process monitoring of a multiphase flow facility," Control Engineering Practice, vol. 42, pp. 74-88, 2015.

[17] B. C. Juricek, D. E. Seborg, and W. E. Larimore, "Predictive monitoring for abnormal situation management," Journal of Process Control, vol. 11, no. 2, pp. 111-128, 2001. 
[18] K. E. Pilario and Y. Cao, "Process incipient fault detection using canonical variate analysis," in 2017 23rd International Conference on Automation and Computing (ICAC). IEEE, sep 2017, pp. 1-6.

[19] G. Vachtsevanos, F. L. Lewis, M. Roemer, A. Hess, and B. Wu, Intelligent Fault Diagnosis and Prognosis for Engineering Systems. John Wiley \& Sons, Inc., 2006.

[20] X. Zhang, M. M. Polycarpou, and T. Parisini, "A robust detection and isolation scheme for abrupt and incipient faults in nonlinear systems," IEEE Transactions on Automatic Control, vol. 47, no. 4, pp. 576-593, 2002.

[21] J. Harmouche, C. Delpha, and D. Diallo, "Incipient fault detection and diagnosis based on Kullback-Leibler divergence using Principal Component Analysis: Part I,’ Signal Processing, vol. 94, no. 1, pp. 278-287, 2014.

[22] F. Harrou, M. Madakyaru, and Y. Sun, "Improved nonlinear fault detection strategy based on the Hellinger distance metric: Plug flow reactor monitoring," Energy and Buildings, vol. 143, pp. 149-161, 2017.

[23] M. Kano, S. Hasebe, I. Hashimoto, and H. Ohno, "Statistical Process Monitoring Based on Dissimilarity of Process Data," IFAC Proceedings Volumes, vol. 33, no. 10, pp. 231-236, jun 2002.

[24] C. Zhao and F. Gao, "A sparse dissimilarity analysis algorithm for incipient fault isolation with no priori fault information," Control Engineering Practice, vol. 65, pp. 70-82, 2017.

[25] J. Shang and M. Chen, "Recursive Dynamic Transformed Component Statistical Analysis for Fault Detection in Dynamic Processes," IEEE Transactions on Industrial Electronics, vol. 65, no. 1, pp. 578-588, 2018.

[26] W. Ge, J. Wang, J. Zhou, H. Wu, and Q. Jin, "Incipient fault detection based on fault extraction and residual evaluation," Industrial and Engineering Chemistry Research, vol. 54, no. 14, pp. 3664-3677, 2015.

[27] H. Ji, X. He, J. Shang, and D. Zhou, "Incipient fault detection with smoothing techniques in statistical process monitoring," Control Engineering Practice, vol. 62, pp. 11-21, 2017.

[28] W. E. Larimore, "Canonical variate analysis in identification, filtering, and adaptive control," Proceedings of the IEEE Conference on Decision and Control, vol. 2, pp. 596-604, 1990.

[29] — , "Optimal Reduced Rank Modeling, Prediction, Monitoring and Control using Canonical Variate Analysis," IFAC Proceedings Volumes, vol. 30, no. 9, pp. 61-66, 1997.

[30] A. Negiz and A. Cinar, "Statistical Monitoring of Multivariable Dynamic Processes with State-Space Models," AIChE Journal, vol. 43, no. 8, pp. 2002-2020, 1997.

[31] B. C. Juricek, D. E. Seborg, and W. Larimore, "Fault Detection Using Canonical Variate Analysis," Industrial \& Engineering Chemistry Research, vol. 43, pp. 458-474, 2004.

[32] Q. Jiang, S. X. Ding, Y. Wang, and X. Yan, "Data-Driven Distributed Local Fault Detection for Large-Scale Processes Based on the GA-Regularized Canonical Correlation Analysis,” IEEE Transactions on Industrial Electronics, vol. 64, no. 10, pp. 8148-8157, 2017.

[33] Z. Chen, S. X. Ding, T. Peng, C. Yang, and W. Gui, "Fault Detection for Non-Gaussian Processes Using Generalized Canonical Correlation Analysis and Randomized Algorithms," IEEE Transactions on Industrial Electronics, vol. 65, no. 2, pp. 1559-1567, feb 2018.

[34] "Feedback controlled cstr process for fault simulation," 2018. [Online]. Available: https://uk.mathworks.com/matlabcentral/fileexchange/66189-feedback-controlled-cstr-process-for-fault-simulation

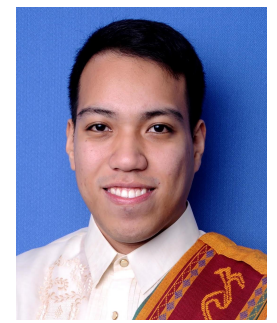

Karl Ezra S. Pilario received his B.Sc. and M.Sc. degrees in Chemical Engineering from the University of the Philippines, Diliman, in 2012 and 2015, respectively. He is currently pursuing a Ph.D. degree at Cranfield University, Cranfield, U.K.

$\mathrm{He}$ is also an Assistant Professor on study leave from the Department of Chemical Engineering at University of the Philippines, Diliman. His research interests are in Process Monitoring and other data-driven methods in Process Systems Engineering. 


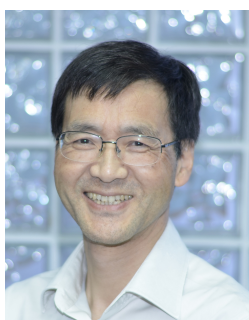

Yi Cao (M'96, SM'10) received the M.Sc. degree in Control Engineering from Zhejiang University, China in 1985 and the Ph.D. degree in Engineering from the University of Exeter, United Kingdom in 1996.

$\mathrm{He}$ is a Reader with the School of Water, Energy and Environment, Cranfield University. His research interests are in Advanced Process Control, including Plant-wide Control, Nonlinear System Identification, Nonlinear Model Predictive Control and Process Monitoring. 
TABLE III

MONITORING PERFORMANCE ${ }^{\dagger}$ FOR THE CSTR INCIPIENT FAULTS

\begin{tabular}{|c|c|c|c|c|c|c|c|}
\hline \multirow{2}{*}{ Fault } & \multirow{2}{*}{$\begin{array}{c}\text { DISSIM } \\
\qquad D\end{array}$} & \multirow{2}{*}{$\begin{array}{c}\text { RDTCSA } \\
D\end{array}$} & \multicolumn{2}{|c|}{ GCCA } & \multicolumn{2}{|c|}{ CVA } & \multirow{2}{*}{$\begin{array}{c}\text { CVDA } \\
\qquad\end{array}$} \\
\hline & & & $T_{r 1}^{2}$ & $T_{r 2}^{2}$ & $T^{2}$ & $Q$ & \\
\hline
\end{tabular}

Multiplicative Faults

\begin{tabular}{cccccccc}
1 & $3.38^{\mathrm{a}}$ & 3.05 & 11.05 & 10.54 & 4.18 & 2.74 & $\mathbf{1 . 4 6}$ \\
& $1.19^{\mathrm{b}}$ & 5.27 & $\mathbf{0 . 0 7}$ & 0.13 & 0.57 & 0.57 & 0.89 \\
& $23.91^{\mathrm{c}}$ & 37.15 & 51.78 & 49.12 & 29.12 & 17.65 & $\mathbf{8 . 9 5}$ \\
\hline \multirow{2}{*}{2} & 5.77 & $\mathrm{ND}$ & 9.47 & 2.95 & 4.82 & 2.63 & $\mathbf{1 . 5 9}$ \\
& 1.40 & 4.41 & $\mathbf{0 . 0 5}$ & 0.12 & 0.59 & 0.64 & 0.93 \\
& 48.05 & 82.62 & 60.05 & 16.81 & 30.26 & 16.49 & $\mathbf{1 0 . 0 2}$ \\
\hline \multirow{2}{*}{3} & 3.21 & 3.36 & 8.33 & 2.93 & 3.39 & 2.42 & $\mathbf{1 . 5 4}$ \\
& 0.76 & 4.35 & $\mathbf{0 . 0 4}$ & 0.09 & 0.46 & 0.53 & 0.75 \\
& 21.45 & 36.28 & 46.03 & 16.49 & 22.57 & 14.96 & $\mathbf{9 . 6 8}$ \\
\hline
\end{tabular}

Additive Faults

\begin{tabular}{|c|c|c|c|c|c|c|c|}
\hline \multirow[t]{3}{*}{4} & ND & 3.75 & 2.33 & 2.30 & 2.00 & 2.35 & 1.44 \\
\hline & 1.03 & 4.90 & 0.07 & 0.11 & 0.52 & 0.67 & 0.83 \\
\hline & 94.62 & 34.73 & 13.54 & 13.27 & 11.48 & 14.45 & 8.62 \\
\hline \multirow[t]{3}{*}{5} & ND & 1.74 & 3.66 & 9.13 & 2.82 & 4.41 & 2.50 \\
\hline & 0.77 & 4.04 & 0.05 & 0.08 & 0.33 & 0.50 & 0.57 \\
\hline & 96.62 & 42.75 & 21.77 & 52.78 & 17.10 & 28.26 & 16.00 \\
\hline \multirow[t]{3}{*}{6} & ND & 3.12 & 8.73 & 3.07 & 3.90 & 2.90 & 2.19 \\
\hline & 0.95 & 5.71 & 0.08 & 0.12 & 0.57 & 0.54 & 0.89 \\
\hline & 96.70 & 58.90 & 51.64 & 17.53 & 23.34 & 18.08 & 13.88 \\
\hline \multirow[t]{3}{*}{7} & ND & 2.60 & 0.41 & 0.38 & 0.36 & 0.23 & 0.15 \\
\hline & 1.10 & 4.01 & 0.07 & 0.11 & 0.55 & 0.52 & 0.83 \\
\hline & 94.41 & 44.93 & 2.22 & 2.07 & 1.96 & 1.31 & 0.82 \\
\hline \multirow[t]{3}{*}{8} & ND & ND & 2.29 & 0.88 & 0.87 & 0.53 & $\mathbf{0 . 3 7}$ \\
\hline & 0.89 & 4.36 & 0.07 & 0.11 & 0.51 & 0.64 & 0.77 \\
\hline & 94.19 & 86.12 & 12.81 & 4.76 & 4.85 & 3.07 & 2.08 \\
\hline \multirow[t]{3}{*}{9} & ND & ND & 6.62 & 0.80 & 1.48 & 0.80 & $\mathbf{0 . 5 3}$ \\
\hline & 1.18 & 5.49 & 0.09 & 0.14 & 0.66 & 0.66 & 1.12 \\
\hline & 93.04 & 76.73 & 37.75 & 4.38 & 8.51 & 4.58 & 3.05 \\
\hline \multirow[t]{3}{*}{10} & ND & ND & 10.51 & 4.35 & 5.22 & 3.38 & 2.37 \\
\hline & 0.87 & 3.98 & 0.06 & 0.09 & 0.34 & 0.50 & 0.60 \\
\hline & 94.75 & 87.39 & 62.10 & 24.74 & 31.73 & 21.11 & 15.01 \\
\hline
\end{tabular}

${ }^{\dagger}$ All results were averaged across 250 faulty data sets monitored in each 